BULL. AUSTRAL. MATH. SOC.

VOL. 7 (1972), 437-441.

\title{
On varieties of soluble groups II
}

\section{J.R.J. Groves}

It is shown that, in a variety which does not contain all metabelian groups and is contained in a product of (finitely many) varieties each of which is soluble or locally finite, every group is an extension of a group of finite exponent by a nilpotent group by a group of finite exponent.

\section{Introduction}

For unexplained notation and terminology and for basic results concerning varieties of groups, we refer to Hanna Neumann's book [7]. We differ from [7], however, in using doubly underlined Romen capitals to represent varieties and $\underline{v}(G)$ to denote the verbal subgroup of the group $G$ corresponaing to the variety $\underline{\mathrm{V}}$.

It has been known for some time now that a proper subvariety $\underline{V}$ of the variety of all metabelian groups is finite exponent by nilpotent by finite exponent; that is, $\underline{V} \leq \underline{B}_{n}{ }_{C} B$ for some integers $n$ and.c (see, for example, 6.1.1 and 6.1.2 of Bryce [1]). It is natural to ask whether this carries over for soluble varieties; more precisely, if $\underline{V}$ is a soluble variety which does not contain $\underline{\underline{A}}^{2}$ - the variety of all metabelian groups - is it true that $\underline{V} \leq \mathrm{B}_{n}^{\mathrm{N}} \mathrm{B}_{n}$ for some integers $n$ and $c$ ? Smel'kin [8] has shown that it is true if $\underline{V}$ is nilpotent by abelian and this was extended by Gupta [4] to the case in which $\underline{V}$ is nilpotent by nilpotent. Also, the present author has shown in [2] that a counterexample to the problem would have to contain a variety which cannot be generated by finite groups.

Received 13 July 1972. The author thanks Dr L.G. Kovács for his constructive suggestions on the presentation of the material. 
The purpose of this note is to give an affirmative answer to the above question. In fact we will prove the following, somewhat more general, result. ${ }^{1}$

THEOREM. Let $\underline{\underline{\mathrm{V}}}$ be a subvariety of a product of (finitely many) varieties each of which is either locally finite or abelian. If $\underline{\mathrm{V}}$ does not contain $\underline{\underline{A}}^{2}$, then $\underline{\underline{\mathrm{V}}} \leq \underline{\underline{B}}_{n} \underline{\underline{N}}{ }_{C}$ for some integers $n$ and $c$.

We observe that this result may be regarded as an extension of a dichotomy of Hall. Using commutator-subgroup functions, Hall defines a special class of varieties of soluble groups - those varieties obtainable from the trivial variety by commutation; see, for example, [5]. He then notes certain natural dichotomies of these varieties; in particular, a 'Hall variety' is either nilpotent or contains the variety of all metabelian groups. The present theorem extends this dichotomy to varieties of soluble groups in general. For a fuller discussion and for a number of related results, we refer to [3].

\section{Proof of the theorem}

The proof will be by contradiction. Let $\underline{v}_{1}$ be a counterexample to the theorem and suppose that $\underline{\mathrm{V}}_{1} \leq \underline{\mathrm{S}}_{1} \ldots \mathrm{S}_{\mathrm{S}}$ where each $\underline{\mathrm{S}}_{i} \quad(1 \leq i \leq r)$ is either locally finite or abelian. Choose $t$ maximal with respect to the property that $\underline{\mathrm{V}}_{2}=\underline{\mathrm{V}}_{1} \wedge \underset{\mathrm{S}_{t}}{\mathrm{~S}} \cdots \mathrm{S}_{\mathrm{r}}$ is still a counterexample; clearly $t<r$. By the choice of $t$, there exist integers $m$ and $d$ such that

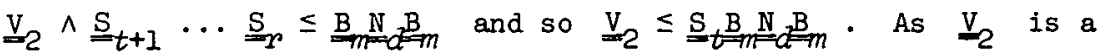
counterexample, it is clear that $\underline{\underline{S}}_{t}=\underline{\underline{A}}$ and that $\underline{\underline{\mathrm{V}}}_{3}=\operatorname{var}\left(\underline{\mathrm{B}}_{m}\left(F_{\infty}\left(\underline{\mathrm{v}}_{2}\right)\right)\right)$ is also a counterexample.

Observe that $\underline{\underline{V}}_{3} \leq \mathrm{AB}_{m} \mathbb{N}$ and let $G=F_{\infty}\left(\underline{\mathrm{V}}_{3}\right)$; let $x$ be a free generator of $G$ and denote $\underset{\rightarrow}{B} N(G)$ by $B$. Then $B$ is abelian and so $\mathrm{BP}(B, x)$ is metabelian. By the hypothesis of the theorem, $8 \mathrm{P}(B, x)$

1 Remark (added 21 August 1972). After this paper was accepted for publication, it was brought to the author's attention that $M_{1}$ l. Kargapolov and V.A. Curkin had already established the soluble case of this result; see $[6]$. 
generates a proper subvariety of $\underline{\underline{A}}^{2}$ and so by, for example, Proposition 3 of [2], $g p(B, x) \in \underline{\underline{B}} \underset{n}{\underline{N}} c \stackrel{B}{=}$ for some integers $n$ and $c$. Hence, if $b \in B,\left[b^{n}, c x^{n}\right]^{n}=1$ (in general, $[g, c h]$ denotes the commutator $[g, h, \ldots, h]$ with $h$ repeated $c$ times). As $B$ is abelian, it follows that $\left[b, c x^{n}\right]^{n^{2}}=1$ for all $b \in B$. Let $T$ denote $\left\{b \in B \mid b^{n^{2}}=1\right\}$. Then $T$ is a fully invariant subgroup of $G$ having finite exponent. As $\underline{\underline{V}}_{3}$ is a counterexample, so also is $\underline{\underline{\mathrm{V}}}=\operatorname{var}(G / T)$. Write $H=G / T=E_{\infty}(\underline{\mathrm{V}})$, denote $x T$ by $y, B / T$ by $A$ and note that, for all elements $a$ of $A,\left[a, c y^{n}\right]=1$.

Let $C$ denote the centraliser of $A$ in $H$; as we noted in [2; p. 98], $C$ is a verbal subgroup of $H$. Hence $K=H / C$ is a relatively free group; let $\left\{y c, y_{1}, \ldots, y_{d+1}\right\}$ be part of a free generating set of $K$ and denote $(y C)^{n}$ by $g$ and $\left[y_{1}, \ldots, y_{d+1}\right]$ by $h$. We claim that $L=\operatorname{gp}\left(h^{g^{i}} \mid i=0,1, \ldots\right)$ is infinite. For, otherwise $h^{g^{j}}=h$ for some integer $j$; rewriting this, $\left[y_{1}, \ldots, y_{d+1},(y C)^{n j}\right]=1$. But this relation in the free generators of $K$ clearly implies that $K \in \mathbb{\mathbb { N }}_{d+1} \stackrel{\mathrm{B}}{n j}_{n j}$. As $K=H / C$ and $C$ centralises $A$, it follows that $\underline{\mathbb{N}}_{d+1} \underline{\underline{B}}_{n j}(H)$

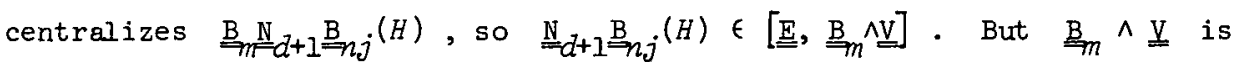
locally finite and so, by Lemma 6 of $[3], \quad[\underline{\underline{E}}, \underline{\underline{B}} \wedge \underline{\underline{V}}] \leq \underline{\underline{B}} q \underline{\underline{A}}$ for some natural number 2 . Hence $\underline{\underline{\mathrm{v}}} \leq \underline{\underline{B}}_{l} \underline{\underline{A N}} d+l \underline{\underline{B}}_{n j}$. However, in this case, it would follow that $\underline{\underline{\mathrm{V}}} \wedge \underline{\underline{A N}} d+1$ is a counterexample which, by Proposition 3 of [2], is not possible. Thus $L$ is infinite; as it is a subgroup of $\stackrel{N}{N}(K)$, which is a group of finite exponent in $y$, it is also locally finite.

Now $K$ is naturally embedded in the automorphism group of $A$ and so also in the endomorphism ring of $A$. In this ring, $(g-1)^{c}=0$ as $a(g-1)^{c}=[a, c g]=1$ for all $a \in A$. Let $M=\operatorname{gp}\left(h^{g^{i}} \mid 0 \leq i<2 c-1\right)$ 
and let $R$ be the subring of this endomorphism ring generated by $M$. As $R$ is additively generated by $M$, the additive subgroup $R^{+}$of $R$ is finitely generated.

The next step is to show that $L \leq R$. It will suffice to show, by induction, that $h^{g^{i}} \in R$ for all $i \geq 0$. This is true by assumption if $i<2 c-1$ and so we suppose that $j \geq 2 c-1$ and that $h^{g^{i}} \in R$ for all $i<j$. A little notation is required. Denote the lie commutator $u v-v u$ of two arbitrary elements $u$ and $v$ of the endomorphism ring of $A$ by $\langle u, v\rangle$; if $k$ is a positive integer, the element $\langle u, k v\rangle$ is just $\langle u, v, \ldots, v\rangle$ with $v$ repeated $k$ times. Also $\left(\begin{array}{l}k \\ i\end{array}\right)$ denotes the binomial coefficient in the usual way. Then the following formula is well known (and easily verified by induction):

$$
\langle u, k v\rangle=\sum_{i=0}^{k}(-1)^{i}\left(\begin{array}{l}
k \\
i
\end{array}\right) v^{i} u v^{k-i} .
$$

Using this formula, it follows that

$$
\begin{aligned}
\sum_{i=0}^{j}(-1)^{j-i}\left(\begin{array}{l}
j \\
i
\end{array}\right) h^{g^{i}} & =g^{-j} \sum_{i=0}^{j}(-1)^{j-i}\left(\begin{array}{c}
j \\
j-i
\end{array}\right) g^{j-i} h g^{i} \\
& =g^{-j}\langle h, j g\rangle=g^{-j}(h, j(g-1)\rangle \\
& =g^{-j} \sum_{i=0}^{j}(-1)^{i}\left(\begin{array}{l}
j \\
i
\end{array}\right)(g-1)^{i} h(g-1)^{j-i} \\
& =0,
\end{aligned}
$$

as $(g-1)^{c}=0$ and, as $j \geq 2 c-1$, either $i \geq c$ or $j-i \geq c$. We can therefore express $h^{g^{j}}$ as a linear combination of the $h^{g^{i}}$ with $i<j$ and so $h^{g^{j}} \in R$, which completes the inductive step. Thus $L \leq R$.

Since $R$ is a ring with identity, its right regular representation gives a natural embedding of itself into the endomorphism ring of $R^{+}$. Hence, by the previous step, $L$ can be embedded as an infinite periodic subgroup of the automorphism group of $R^{+}$. But $R^{+}$is a finitely 
generated abelian group and it is well known (see, for example, Theorem TI of Wehrfritz [9]) that every periodic subgroup of its automorphism group is therefore finite. This contradiction completes the proof of the theorem.

\section{References}

[1] R.A. Bryce, "Metabelian groups and varieties", Philos. Trans. Roy. Soc. London Ser. A 266 (1970), 281-355.

[2] J.R.J. Groves, "On varieties of soluble groups", Bull. Austral. Math. Soc. 5 (1971), 95-109.

[3] J.R.J. Groves, "Varieties of soluble groups and a dichotomy of P. Hall", Bulz. Austral. Math. Soc. 5 (1971), 391-410.

[4] Narain Gupta, "On metanilpotent verieties of groups", Canad. J. Math. $22(1970), 875-877$.

[5] P. Hall, "Finiteness conditions for soluble groups", Proc. London Math. Soc. (3) 4 (1954), 419-436.

[6] M.H. Каргаполов, B.А. Чурнин [M.।. Kargapolov, V.A. Čurkin], "0 многообраэнях разрвшимых групп" [On varieties of soluble groups], Algebra i Logika 10 (1971), 651-657.

[7] Hanna Neumann, Varieties of groups (Ergebnisse der Mathematik und ihrer Grenzgebiete, Band 37. Springer-Verlag, Berlin, Heidelberg, New York, 1967).

[8] A.L. Smel'kin, "On soluble groups yarieties", Soviet Math. Dokz. 9 (1968), 100-103.

[9] B.A.F. Wehrfritz, "Groups of automorphisms of soluble groups", Proc. London Math. Soc. (3) 20 (1970), 101-122.

University of Manitoba,

Winnipeg, Canada.

Present address:

Department of Mathematics,

University of Melbourne,

Parkville,

victoria. 